\title{
InnoJudS: Web-Based Innovation Judging System
}

\author{
R.A.JM.Gining ${ }^{1}$, S.S.M.Fauzi ${ }^{2}$, T.R.Razak ${ }^{3}$, M.N.F.Jamaluddin ${ }^{4}$, N.A.A.Zahari ${ }^{5}$ \\ ${ }^{1}$ Faculty of Computer and Mathematical Sciences, UniversitiTeknologi MARA, Perlis Branch, Malaysia, \\ raysocialemail@gmail.com \\ $2^{2 *}$ Faculty of Computer and Mathematical Sciences, UniversitiTeknologi MARA, Perlis Branch, Malaysia, \\ shukorsanim@uitm.edu.my \\ ${ }^{3}$ Faculty of Computer and Mathematical Sciences, UniversitiTeknologi MARA, Perlis Branch, Malaysia, \\ tajulrosli@uitm.edu.my, \\ ${ }^{4}$ Faculty of Computer and Mathematical Sciences, UniversitiTeknologi MARA, Perlis Branch, Malaysia, \\ nabilfikri@uitm.edu.my \\ ${ }^{5}$ Faculty of Computer and Mathematical Sciences, UniversitiTeknologi MARA, Perlis Branch, Malaysia, \\ ray_adderley@uitm.edu.my
}

\begin{abstract}
The paper aims to develop InnoJudS: Web-Based Innovation Judging System based on the previously gathered requirement in [7]. Works of literature related to a Judging System approaches are examined to confirm the notion of developing a system is better compared to the manual approach. Laravel Framework is utilised to assist the development process of the proposed system. The methodology adopted divides into three phases; interface design, database creation and coding phase each producing results. The first phase produces sitemaps and the rough sketches of the user interface to be implemented in the system. Followed by the database creation phase which produces database based on the ERD requirement in [7]. The final phase is supported by Laravel Framework with its MVC elements (Model, View and Controller); interpreted the user interface in the first phase as HTML file (View), the created database into Models and the functionalities requirement in [7] as Controller functions. The final phase completes the development of InnoJudS.
\end{abstract}

Key words: System development, web system, judging system, Laravel framework, MVC framework

\section{INTRODUCTION}

Innovation is a broad field. In "Oslo Manual", innovation is the application of a new or notably upgraded product which is service or good, procedure, new marketing technique or a new organizational method either in workplace organization, business practices or external relation. To live in a turbulent surrounding, big organizations need to innovate its goods, processes and services continuously. Consistent with the needs, producing radical or advance innovations could be achieved by competition-based innovation. Therefore, many innovation competitions are organized widely by different institutions to encourage new product development [1-3].
Every innovation competition required judging process conducted by judges to evaluate the innovated products. Typically, at least two judges comprise the field expert and non-field expert are assigned as judges for a product which are done weeks before the competition to ensure fairness. During the competition, the judges need to identify themselves to the secretariat and to get a briefing from the Head of Judge. Subsequently, the judges then need to visit and evaluate all the product situated in booths assigned to them. The evaluation is done by filling in the prepared form and return it to the secretariat after. After the forms are received, the data needs to be transferred to a chosen platform; usually, Microsoft Excel, which used to sort the scores to identify the winner.

The mentioned process leads to several concerns and drawbacks. Firstly, the judges have multiple evaluation form, which is cumbersome to hold and manage. The probability of the evaluation form misplaced, damaged and lost is also present. Secondly, inserting data from evaluation form to Microsoft Excel consume much time and can cause a person to lose focus. If this situation continues, a massive delay in announcing the winners in innovation competition inevitably will occur. Thirdly, other than demanding more time and resources, manual data entry is also burdensome. The process of transferring the data from the form to a Microsoft Excel can lead to a few problems such as typographical error, incorrect placement of data, misinterpretation and entered false data could happen. The data entry from physical to electronic undertaken by humans is a slow and repetitive process which leads to bleary and fatigue[4-5].

Hence, to improve the judging process, manual data entry must be lessened or possibly eliminated. Prior studies suggest internet-based data gathering can avoid many hours of data entry if utilised correctly [6]. This study proposes to develop a Web-based Innovation Judging System (InnoJudS) based on the previously gathered requirement in [7]. The developed system is expected to 
assist the innovation competition organizer in managing its judging process effectively.

\section{LITERATURE REVIEW}

This section describes the literature reviews relevant to the development of the proposed system. These comprise the study on available judging approaches, Laravel framework and the previous work identified requirement.

\subsection{Judging System Approaches}

Two approaches are highlighted, the manual and the webbased approach of the judging process. In the manual approach, the judges need to fill in the evaluation forms provided by the competition secretariat by hand. This means the judges have to bring multiple forms for the respective products assigned to them for evaluation. The form usually contained necessary information, and it possessed unique characteristics to help the judges identify their assigned product. Usually, the evaluation rubric is also provided together with the form as guidance. This leads to a collection of forms that need to be managed by the judges, which are cumbersome.

Nevertheless, this approach has a few advantages. The first advantage is its complexity. Manual data entry is closely related to a file where all the data are stored. A traditional file approach is less complicated compare to electronic systems [8]. For example, it is easier for an untrained person to access and manipulate data. Different from managing electronic database information that may involve technical skills - a user mistake can lead to data loss or unintentional alterations. The second advantage is accuracy. The most crucial in data entry is the accuracy of data inserted into a computer. Manual data entry has the probability of being more accurate compared to the automated data entry. An employee can identify and correct mistakes as they happen. So a focused data entry employee may make fewer mistakes than an automated system [5].

However, manual data entry does introduce the perchance of human error. Employees that do not have excellent attention to detail or that are suffering from fatigue or lack of concentration may increase the number of mistakes. This points to the disadvantages of the manual approach that is human error. A simple human error, like the data input problems, although a minor typographical error, can lead to short- and long-term problems. This error is leading to misinformation, disorganization, and inaccurate records. Another disadvantage is the cost. To type a single page of information into a computer can take up to several minutes which are time-consuming. The cost of purchasing necessary equipment and software to enter data into a computer automatically is much lower in price than the cost of hiring data entry workers especially if huge totals of information need to be transferred into a digital state [9].
Meanwhile, a web-based approach is employing any applications that utilize a website as the front-end or interface is known as a web-based application. By using a standard browser, users can use the application from any types of computer that connected to the preferred network. This allows the judging process to be done electronically and its data to be managed in one centralized database server.

Accessibility is one of the benefits. Different from traditional applications, web systems are available anywhere and anytime through any computer with an Internet connection [10]. This also creates modern, exciting possibilities such as real-time collaboration, global teams and home working. With web-based applications, the idea of sitting while facing a computer and working in an exact location is no longer happen. Another advantage is related to installation and maintenance, where it becomes less complicated with a web-based approach. There is no need to upgrade the application in eachof the user computers for latest version installation. All users can access the latest version thoroughthe host server where the update is centralized. In the innovation competition context, all the data could enter directly by the judges into a centralized database which then can be retrieved by the secretariat without the hassle of getting the data from the judges through a standard form.

Along with all the advantages, there are also a few disadvantages of the web-based approach. As an example, the issue such as the difficulty of end-user adoption towards the web-based approach, which requires training. In the competition judging context, the judges might not be accustomed to the web-based approach, thus a comprehensive briefing on the system usage needed to be arranged[11].

\subsection{Laravel Framework}

In this study, to assist and eases the development process, the proposed system utilises the Laravel framework. According to [12], Laravel framework is based on a multi-layer structure which is a free, open-source web application framework that uses PHP programming language. Some of the Laravel features is that it involves eloquent ORM, which is an advanced PHP implementation that provides constraints for the relationship between databases. It also uses the migration concept for database management. Laravel is the preferable PHP framework as it took up to $26 \%$ place in the development of web application [13].

The Laravel framework exercises the application of Model, View, and Controller (MVC) architecture. MVC is an architecture which has three-layer of development for a web application [14]. Developers widely use it as a standard design pattern which provides a complete framework for the developers. The primary purpose of using MVC is to reduce the gap between digital and human model [15]. 


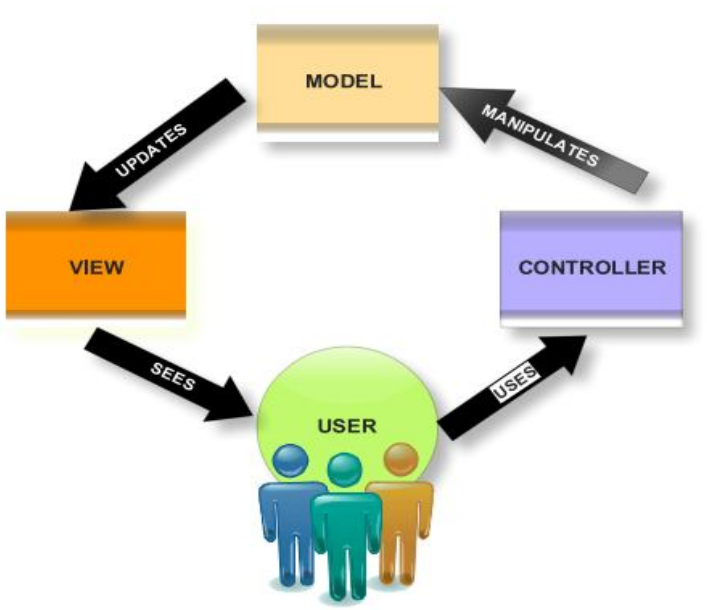

Figure 1: MVC architecture [13]

One of the layers is the Model layer which is used to manage tasks that are related to data access abstraction as well as validation. It is also used to insert, retrieve and update the data in the database - the business logic of the system. The View layer is responsible for the interface of the application. This layer is also known as web design or templates. It is responsible for the way data is displayed and on how the user interacts with the application. Lastly is the controller layer. The controller layer is for managing event handling [15]. It means that the controller is responsible for handling the user's request by interacting with the Model and the View [14].

Overall, it may be said that MVC architecture makes the development process faster and simpler. The modification of the proposed system will be more straightforward since there is a separation between the operations.

\subsection{System Requirement}

The proposed system is developed based on the requirement identified in the previous work in [7]. This is a significant process as mentioned in [16] where it focuses on end-user requirement gathering without any biases. The requirement identification methodology consists of two phases; gathering and analysis. The requirement gathering phase consists of two main activities which are interview and document review. The output of the activities is a list of requirements sorted into two types - functional and non-functional, which further sorted based on priorities.

Table 1: Gathered functional requirements sample[7]

\begin{tabular}{|l|l|l|l|}
\hline \multicolumn{4}{|c|}{ A. FUNCTIONAL REQUIREMENTS } \\
\hline No. & Req ID & \multicolumn{1}{|c|}{ ReqDescription } & Priority \\
\hline & JS_01 & $\begin{array}{l}\text { Login into the system (Judge } \\
\text { and Admin) }\end{array}$ & \\
\hline 1. & $\begin{array}{l}\text { JS } \\
\text { _01_01 }\end{array}$ & $\begin{array}{l}\text { Judge and admin must enter } \\
\text { email and password to login. }\end{array}$ & M \\
\hline 2. & $\begin{array}{l}\text { JS } \\
\text { _01_02 }\end{array}$ & $\begin{array}{l}\text { Message will be prompted if } \\
\text { email and password do not }\end{array}$ & D \\
\hline
\end{tabular}

\begin{tabular}{|l|l|l|l|}
\hline & & match. & \\
\hline 3. & JS & $\begin{array}{l}\text { Could click on Forgot Your } \\
\text { Password if the user forgot } \\
\text { their password. }\end{array}$ & 0 \\
\hline
\end{tabular}

These requirements then used as the input in the second phase of requirement analysis. Several analysis tools are used in the analysis phase like Use Case Diagram, Activity Diagram, and Entity Relationship Diagram (ERD). The figure below shows the Use Case Diagram developed from extracting useful information from the output in the first phase.

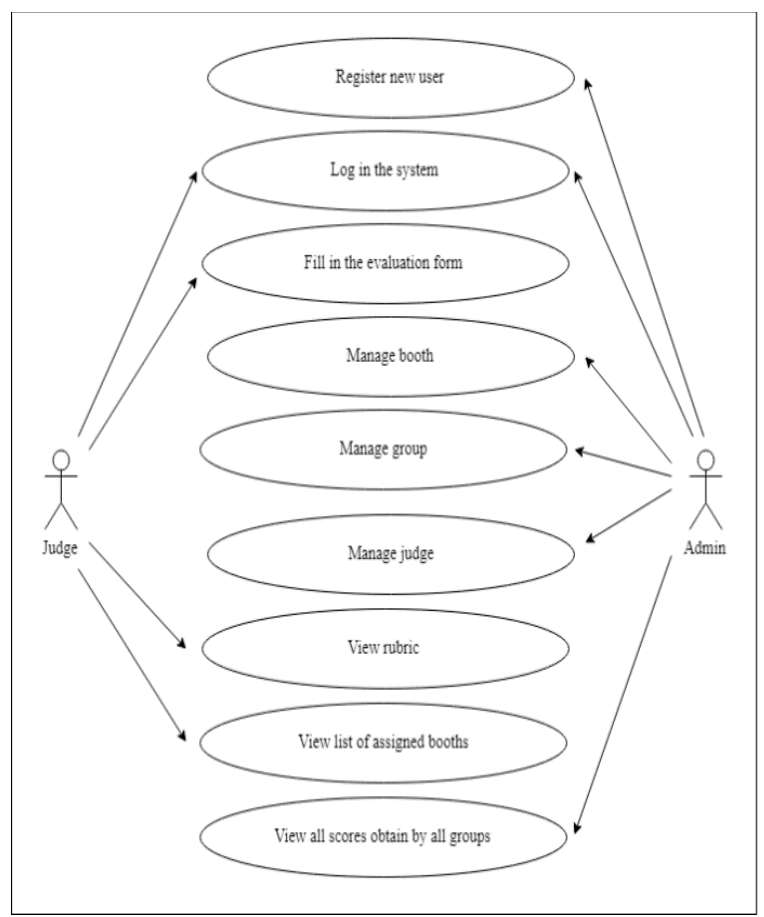

Figure 2: UCD from Requirement Analysis [7]

\section{METHODOLOGY}

The methodology used in the development of the proposed system involves three phases; interface design, database creation and programming or coding. Each phase implements the information gathered in the previous works towards the completion of the proposed system.

\subsection{Interface Design Phase}

In the first phase, the entire interface of the system is designed by using sitemap and lo-fi prototype; the storyboard. The sitemap is produced as the user interface page navigation. Storyboarding is a part of the initial stages, which serves as a blueprint for the system. It is a simple, flexible tool which can be used to display the elements on a single web page such as images, banners, navigation, graphic elements and text. The purpose of this storyboard is that it serves as an outline of the design approach, defines the elements that need to go on each page. It illustrates the navigational architecture and information flow. Also, it demonstrates how the pages are 
to work together to provide the user's interactive experience.

\subsection{Database Creation Phase}

In the second phase, the database creation and management - MySQL is being used. MySQL runs on nearly all platforms, including Linux, Windows and UNIX. MySQL usually related to web applications and online publishing even though it can be used in a wide range of applications [17]. Another alternative is choosing the NoSQL database [18] but in the case of Laravel Framework, MySQL is utilized. MySQL provides features such as; create, copy, browse, drop, rename and alter database, tables, fields and indexes, maintenance server, databases and table, with a proposal on server configuration, and execute, edit and bookmark any SQL-statement including batch query. Additionally, the use of the Laravel framework migration features further supports the management of the system database.

\subsection{Coding / Programming Phase}

In the last phase of coding or programming, to provide a solid foundation for the proposed system, the use of the Laravel framework is employed. Laravel allows the developer to transform a concept into a working prototype rapidly. It aims to remove the pain in development by facilitating the common tasks used in most web projects, for instance, verification, routing, caching and sessions [15]. Developers can create their infrastructure that is specifically designed for their application because Laravel has a unique architecture. To write the coding, PhpStorm Integrated Development Environment (IDE) is used. PhpStorm is a full-featured, cross-platform PHP IDE built on JetBrains' IntelliJ IDEA. It offers all features and tools for PHP and supports Front-end technologies. It was aimed to assist the development of Internet applications written in PHP.

By referring to the interface design, the View layer of the system in MVC architecture is produced. Further supplanted by the combination of database creation and the coding phase, which mostly incorporates the Model and Controller layer - the proposed system is developed complying firmly with the identified requirement in [7].

\section{RESULT AND DISCUSSION}

The result of the first phase activities are translated into a sitemap, and the user interface designs. These are produced from referring to the Use Case Diagram presented in the previous work. The system consists of two types of users with several accessible functions which translated into the sitemap. The sitemap shows the navigation structure of the system in the hierarchical form. From home, the user needs to go to the login page to log into their account either as judge or admin. As a judge, they only can view the list of assigned booths and access the evaluation form for each booth. As an admin, they can navigate through the system using Group, Booth, Report and Register New User menus. Some of the menus have a sub-menus which provides the admin capabilities to manage the information related to the specific menu. The figure below shows the full sitemap for the system based on the requirement gathered in the previous work in [7].

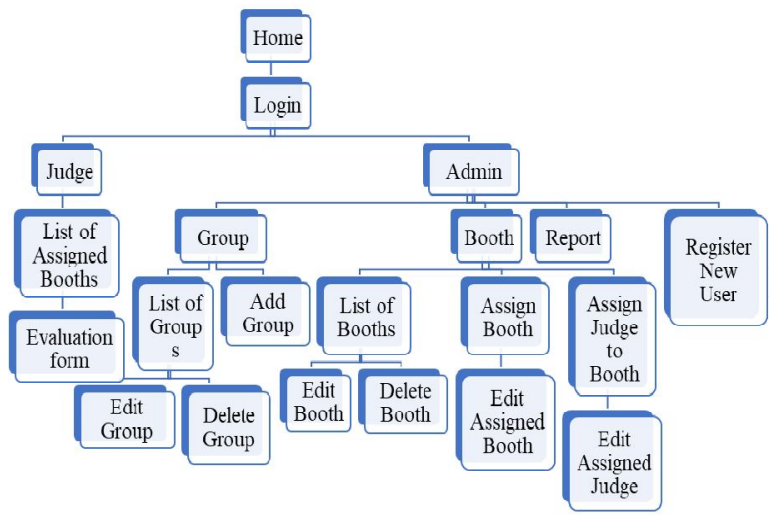

Figure 3: Sitemap of InnoJudS

The user interface design which represented by storyboard is used to illustrate the navigational architecture and information flow to present an interactive experience in each of the system pages. In the figure below, it shows the storyboard for the login page of the proposed system. Both admin and judge can log in into the system through this page.

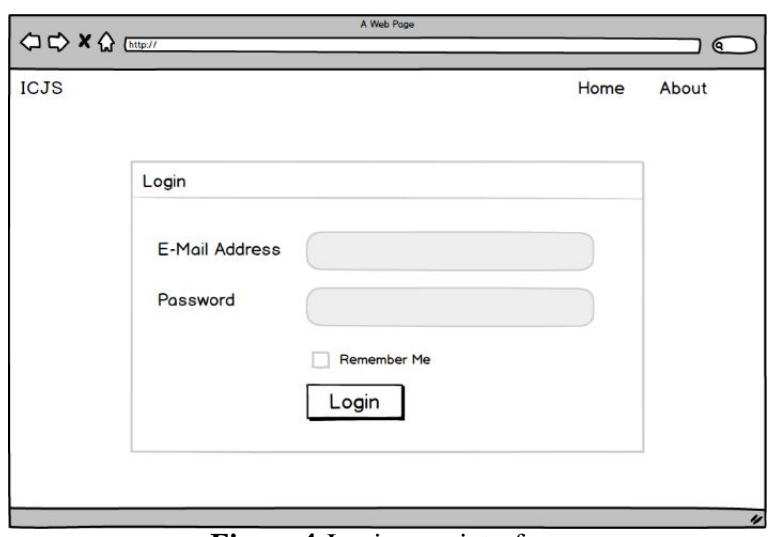

Figure 4:Login user interface

The next storyboard figure below illustrates the evaluation form for the judges to use. This evaluation form is displayed when the judge clicks on the booth number that already assigned to them (figure 5).

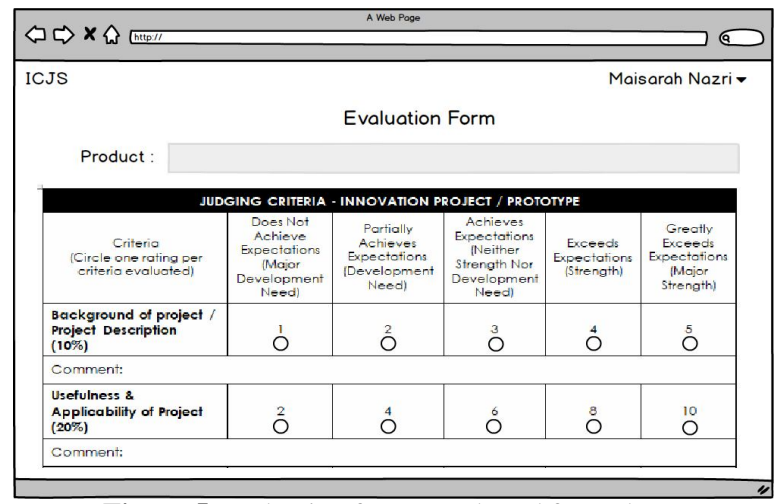

Figure 5:Evaluation form storyboard for Judges 


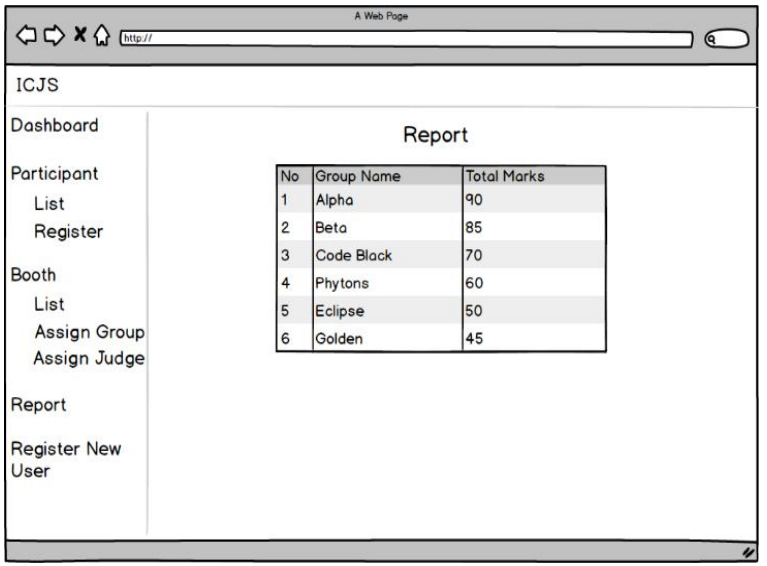

Figure 6:Result report for Admin reference

Figure 6 above shows the storyboard of the report of the total marks for each group asa reference for the admin. The list of this group is displayed according to the marks obtained by each group sorted in descending order.

The second phase constructs the database by utilising phpMyAdmin software which supports MySQL features for database management. The requirement translated from the previous work Entity Relationship Diagram (ERD). The previous requirement provided nine entities (booths, booths_users, groups, group_members, products, product_types, criteria, criteria_scales, scales and users).

The entities in the ERD are interpreted as tables, and the characteristics of it became the attributes of the table in the produced database. The migration features of the Laravel framework also play a crucial role in completing the database through structuring and seeding process. Figure 7 below shows the completed database structure through phpMyAdmin software. There are supposed to be nine tables matching the nine entities. However, there are three additional tables (migrations, password_resets and roles) which are required to enable the use of migration features of the Laravel framework.

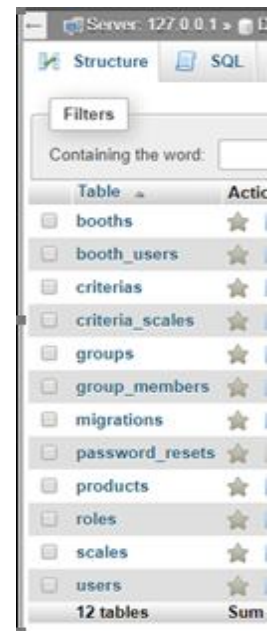

Figure 7:List of tables translated in MySQL database from previous work ERD
The final phase of the methodology, coding - achieves the complete development of the proposed system. As described in the methodology section, the Laravel framework and the PhpStorm IDE are the technologies operated mostly throughout the final phase. The ModelView-Controller (MVC) architecture which enabled by the Laravel framework, is implemented in this phase.

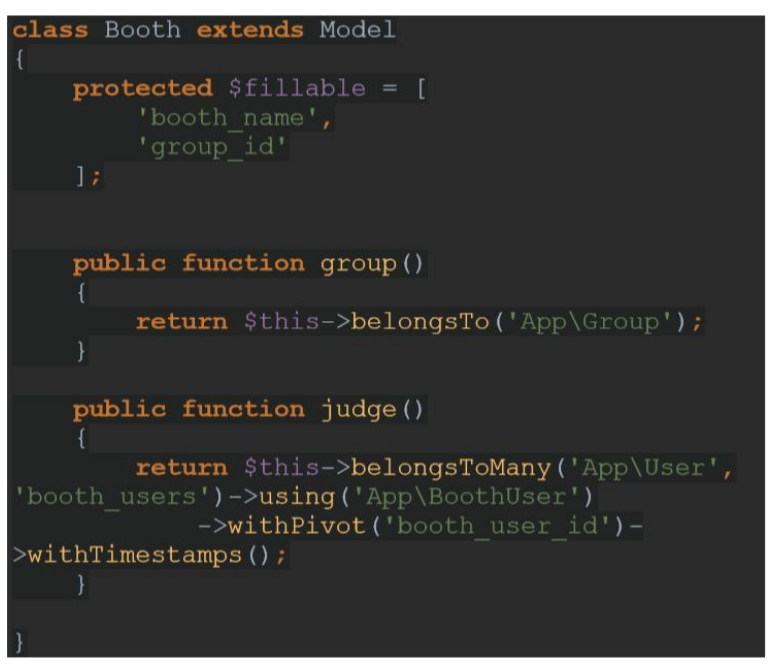

Figure 8:Booth Model code snippet

The figure aboveis the code snippet for the Booth Model implemented for the booths assigned to the judge for evaluation. The Model in the MVC architecture reflects the tables generated in the database. The attributes of the booths can be seen available in the \$fillable attribute of the model. The functions written in the Model acts as a relationship between the Booth Model to the other models. As an example, by referring to the Judge function inside the Booth model, these two models have a ManyTo-Many relationship towards each other.

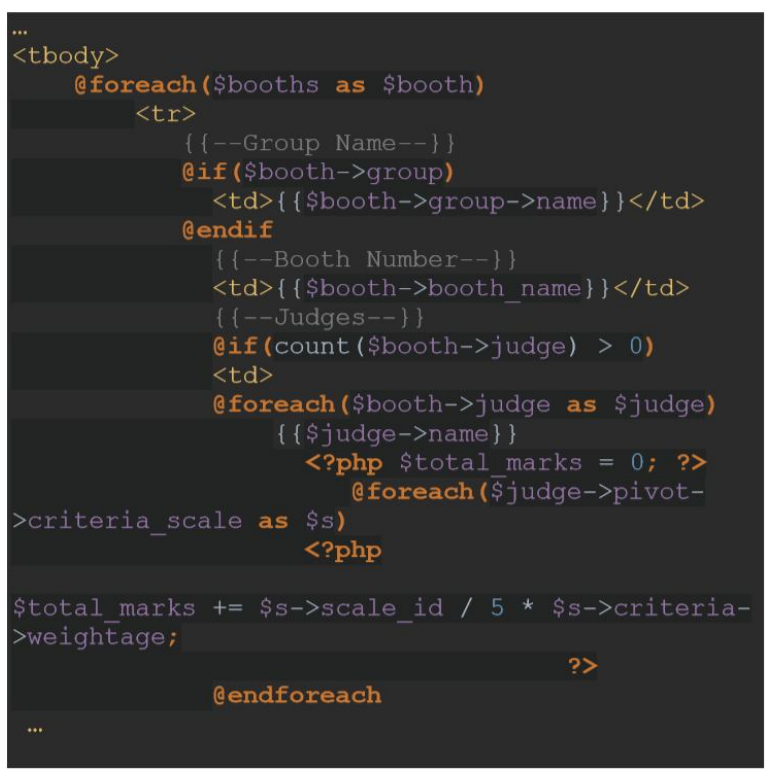

Figure 9: Report View code snippet 
The Controller accessed the Model to manage the data from the database, then passing the accessed data to a specific View if necessary. As an example, the simple code snippet below shows the report Controller. The controller starts with accessing the Booth model, retrieving all the Booths data using the all() function. The data is stored in the \$booths attribute then passed to the View 'staff.admin.report' in the subsequent line.

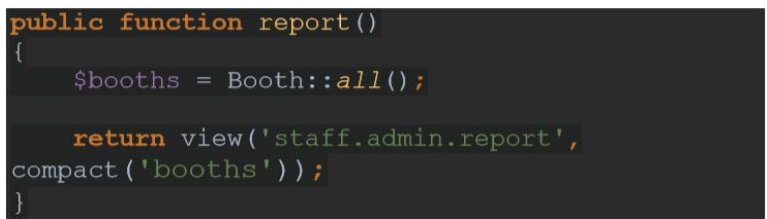

Figure 10: Report Controller code snippet

Other than the layers of the MVC architecture, the created Route of the system is also an essential component of the system. The Route functions as a mapper of the requests relayed to the system. Necessary routing routes the relayed request to the relevant controllers. Figure 11 below shows the route created for the report Controller described in figure 10. The method used is the get() method which used for data retrieval purposes. Meanwhile, the '/admin/report' is the Uniform Resource Identifier (URI) used to access the route through the web browser. This route then utilises the ScoreController specifically the report() function declared in 'ScoreController@report' and applied the 'staff.admin.report' as the name of this created route.

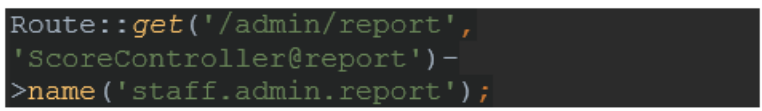

Figure 11: Route for the report Controller

The combination of these essential components composes the complete proposed system. With the completion of the system, it is expected to run as per the requirement identified in the previous work.

\section{CONCLUSION}

This paper described a study on the design and development of the proposed web-based innovation judging system (innoJudS). The requirements implemented in the system are based on the previous work of requirement identification performed in [7]. The methodology applied in this study consists of three phases which translate the identified requirements into designs (sitemap and user interface), database and business logic which produce the completed proposed system.

\section{REFERENCES}

1. Obradovic, D., \&Obradovic, D. (2016). The Role Innovation on Strategic Orientations and Competitiveness of Enterprises. Ecoforum, 5(1).

2. Hossain, M., \&Kauranen, I. (2014). CompetitionBased Innovation: The Case of the $X$ Prize Foundation. Journal of Organization Design, 3(3). https://doi.org/10.7146/jod.9790
3. Yang, Y. (2012). Open Innovation Contests In Online Markets: Idea Generation And Idea Evaluation With Collective Intelligence A Dissertation Submitted to the Temple University Graduate Board in Partial fulfillment of the Requirements for the Degree of Doctor Of Philosophy. In Dissertation.

4. Vlada, M., Babiy, I., \&Ivanescu, O. (2010). ABBYY recognition technologies - ideal alternative to manual data entry - Automating processing of exam tests .Star, 3(1), 3-8.

5. Hamel, G. (2017). Advantages \& Disadvantages to Manual Data Entry. http://www.ehow.co.uk/info_8585434_advantagesdisadvantages-manual-data-entry.html

6. Benfield, J. A., \&Szlemko, W. J. (2006). Internetbased data collection: Promises and realities. Journal of Research Practice.

7. Zahari, N. A. A., Gining, R. A. J., Fauzi, S. S. M., Razak, T. R., \& Jamaluddin, M. N. F. (2020). An Identification of Software Requirement for Web Based Judging System. IOP Conference Series: Materials Science and Engineering, 767, 012033. https://doi.org/10.1088/1757-899X/767/1/012033

8. Hamel, G. (2018). Advantages \& Disadvantages of Traditional File Organization. Small Business. https://smallbusiness.chron.com/advantagesdisadvantages-traditional-file-organization41400.html

9. Chao, C. (2015). Implementing a Paperless System for Small and Medium-Sized Businesses ( $S M B S$ ). 1277(December).

10. Qu, Z., Ninan, S., Almosa, A., Chang, K. G., Kuruvilla, S., \& Nguyen, N. (2007). Synoptic reporting in tumor pathology: Advantages of a web-based system. American Journal of Clinical

11. James ye. (2009). Issues AndDisadvatages Of Moving To A Paperless Office James Nye. In Issues and disadvantages of moving to paperless office. Issues.

12. He, R. Y. (2015). design and implementation of web based on laravel framework. Proceedings of the 2014 International Conference on Computer Science and Electronic Technology. https://doi.org/10.2991/iccset-14.2015.66

13. Olanrewaju, R. F., Islam, T., \& Ali, N. (2015). An Empirical Study of the Evolution of PHP MVC Framework. In Lecture Notes in Electrical Engineering (Vol. 315, pp. 399-410). https://doi.org/10.1007/978-3-319-07674-4_40

14. Majeed, A., \& Rauf, I. (2018). MVC Architecture: A Detailed Insight to the Modern Web Applications Development. Peer Review Journal of Solar \& Photoenergy Systems, 1(1).

15. Pop, D. P., \& Altar, A. (2014). Designing an MVC model for rapid web application development. Procedia Engineering, 69, 1172-1179. https://doi.org/10.1016/j.proeng.2014.03.106

16. Dalal, S. (2019). Exploring the Essentials and Principles of Software Development. International Journal of Advanced Trends in Computer Science and Engineering, 8(6), 3504-3510. https://doi.org/10.30534/ijatcse/2019/129862019 
17. Stephens, J., \& Russell, C. (2004). Beginning MySQL Database Design and Optimization. In Beginning MySQL Database Design and Optimization. Springer. https://doi.org/10.1007/9781-4302-0729-0

18. Banane, M., \&Belangour, A. RDFMongo: A MongoDB Distributed and Scalable RDF management system based on Meta-model. International Journal of Advanced Trends in Computer Science and Engineering, 8, 734-741. https://doi.org/10.30534/ijatcse/2019/62832019 